\title{
Initial assessment of framework for delivery of elective orthopaedic surgery in the United Kingdom following the COVID-19 pandemic
}

\author{
S. Sharkey ${ }^{1}$ (1) $\cdot$ K. Narang ${ }^{1} \cdot$ R. J. Pacheco ${ }^{1} \cdot$ C. Anthony ${ }^{1}$
}

Received: 11 January 2021 / Accepted: 1 June 2021 / Published online: 9 June 2021

(C) Istituto Ortopedico Rizzoli 2021

\begin{abstract}
Purpose In light of the COVID-19 pandemic, a framework for safe provision of elective orthopaedic surgery must be developed in order to restore and maintain activity. The aim of this study was to explore patient attitudes to surgery and theatre efficiency as a result of the COVID-19 pandemic and assess a potential framework for the delivery of such services.

Methods Prospectively collected data on theatre timings and procedures completed per session used to assess theatre efficiency comparing June 2019 to June 2020. Information on patient compliance with 14-day household isolation and attitudes to surgery were collected prospectively over a seven-week period using a questionnaire. Follow-up data were collected via telephone consultation a minimum of two weeks after discharge.

Results Significant reduction in the number of points per session $(p=0.02)$ with a mean of 3.19 in 2019 and 2.42 in 2020. Only 18 of 31 patients were compliant with pre-operative isolation with individual failures accounting for four of 13 and failures by household members accounting for nine. Impact of COVID-19 and precautions on patient anxiety was mixed. No patients required symptomatic COVID-19 swab post-operatively.

Conclusion With the restrictions of COVID-19, there are significant problems with theatre efficiency, in effect losing an operation a list. Furthermore, compliance with pre-operative isolation was poor but to the best of our knowledge no patients became unwell from COVID-19 post-operatively. Additional strategies will be required to reinstate an effective elective orthopaedic service, especially as the nation heads into another wave.
\end{abstract}

Keywords COVID-19 Orthopaedics Elective surgical procedures $\cdot$ Severe acute respiratory syndrome coronavirus 2

\section{Introduction}

The world health organisation (WHO) announced a global pandemic on 11 March 2020 advising all countries to undertake "urgent and aggressive action" in response to the rapidly spreading 2019 novel coronavirus disease, termed COVID-19 [1,2]. Due to the rate at which COVID-19 infection spread throughout the population, including amongst healthcare workers, the National Health Service (NHS) in the UK was forced to take unprecedented action in response to the pandemic. From 15 April 2020, all elective surgery, with the exception of urgent cancer surgery, ceased and theatres were prepared for use as critical care areas as instructed by NHS England as part of a national directive. This action

S. Sharkey

samanthasharkey@ doctors.org.uk

1 Trauma and Orthopaedics Department, Goole and District Hospital, Goole DN14 6RX, England, UK also allowed preservation of personal protective equipment (PPE) $[3,4]$.

Our orthopaedic service is made up of a separate trauma site and elective site, the latter of which has since been transformed into a "clean" COVID-19 free site. Elective orthopaedic lists were re-introduced at this clean site in June 2020 on the provision that all patients are risk-stratified prior to listing, complete a strict 14-day isolation period, along with their household and must have had a negative COVID-19 swab within $72 \mathrm{~h}$ of their operation. In accordance with Public Health England (PHE) guidance, staff onsite are required to wear PPE and there is a $20 \mathrm{~min}$ 'dead time' from the patient exiting the operating theatre before cleaning the theatre in preparation for the next patient.

It was our aim to assess the impact of new infection control protocols on theatre efficiency by comparing the number of cases completed per session in June 2020 with that in June 2019. We also aimed to assess patient compliance with household isolation and attitudes towards surgery with 
the introduction of new precautions and additional risk. The null hypotheses were that patients would be fully compliant with isolation and there would be no difference in attitudes towards surgery nor theatre efficiency as a result of the COVID-19 pandemic.

\section{Material and Methods}

\section{Theatre efficiency}

All elective orthopaedic cases performed within the study period were included. There were 40 cases identified in June 2020 and 85 cases identified in June 2019. Theatre timings were collected prospectively using an electronic clinical software system which was accessed in a retrospective manner. Theatre lists were populated using a standardised points system based on expected case length as illustrated in Table 1 and number of points per session calculated. Assessment of data normality was undertaken with Shapiro-Wilk test, parametric data were analysed with a 2-tail t-test and nonparametric data with a Mann-Whitney U test. Data were deemed statistically significant with an alpha value of less than 0.05 .

\section{Patient compliance and attitudes to surgery}

All patients attending for elective orthopaedic procedures over a seven-week period in June/July 2020 were invited to take part in this single centre study. Initial data were collected prospectively in the form of a questionnaire completed by the patient and the operating team. A Fisher's exact test was used to determine whether patients with risk factors for COVID-19 related complications were significantly more likely to remain compliant with pre-operative isolation or experience increased anxiety about their upcoming surgery when compared to those with no risk factors.

Follow-up data relating to complications were obtained via telephone consultation using a pre-defined set of questions a minimum of 14 days after discharge. All patients were asked about the following symptoms to screen for postoperative COVID-19; cough, fever, loss of taste or smell, diarrhoea, fatigue, myalgia and headache. Of 58 patients attending during this time period, 37 (63.8\%) were included in this study with follow-up data obtained for $33(89.2 \%)$ of these patients (see Fig. 1 for details).

\section{Results}

\section{Theatre efficiency}

There was a significant reduction in the number of points per session $(p=0.02)$ with a mean of 3.19(CI 2.71-3.67) in 2019 and 2.42(CI 1.87-2.97) in 2020. Each stage prior to and following the procedure was analysed to identify whether this was due to a global delay or associated with any specific stage and the results are found in Table 2. Of note, there was a significant increase in the time from finishing procedure to patient leaving the operating theatre of two minutes(CI 1-4) with $\mathrm{p}$ value 0.006 and from time of leaving the operating theatre to sending for the next patient of $43 \mathrm{~min}$ (CI 32-47) with $p$ value $<0.0001$. There was no significant difference in the time taken from sending for patient to arriving at theatres, from arriving to entering operating theatre or from entering operating theatre to starting procedure.

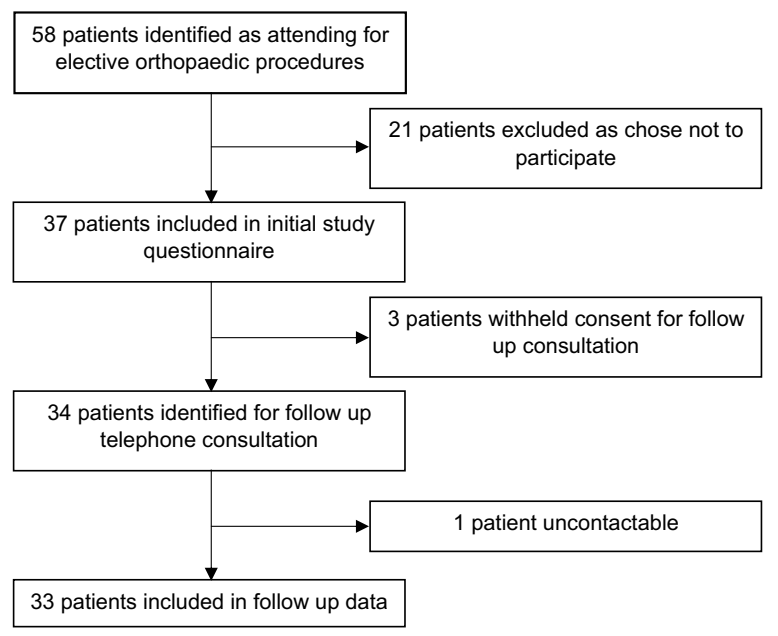

Fig. 1 Flow chart of patients' inclusion and exclusion. Patients identified as eligible during 7 week study period $(n=58)$. Exclusions included patients who opted out during consent process $(n=21$ in initial phase, $n=3$ for follow-up consultation), or uncontactable for follow-up consultation $(n=1)$

Table 1 Points system used for standardisation

\begin{tabular}{llll}
\hline 3 points & 2 points & 1 point & 0.5 points \\
\hline Revision arthroplasty & Joint arthroplasty & Knee arthroscopy & Carpal tunnel decompression \\
& ACL reconstruction & Shoulder ASAD & Injection to joint (including under X-Ray) \\
& MPFL construction & Dupuytren's contracture release & Trigger finger/thumb release \\
& Rotator cuff repair & Decompression of ulnar nerve & \\
& Ankle fusion & Hallux valgus correction & \\
\hline
\end{tabular}


Table 2 Comparison of theatre timings from 2019 to 2020: Mean time taken transitioning from each of the recorded stages illustrated with $95 \%$ confidence intervals. Comparisons made between timings in 2019 (i.e. prior to COVID-19 pandemic) and 2020 (following institution of new COVID-19 protocols)

\begin{tabular}{llll}
\hline Transition period & Mean time taken (minutes) 2019 & Mean time taken (minutes) 2020 & $P$ value \\
\hline Sent for to arrived at theatre suite & $8.07(7.22-8.92)$ & $9.21(7.49-10.92)$ & $17.51(13.96-21.07)$ \\
Arrived at theatre suite to entered operating room & $16.09(13.41-18.78)$ & $11.13(8.56-13.7)$ & 0.2 \\
Entered operating room to started procedure & $8.92(7.59-10.24)$ & $5.87(4.59-7.16)$ & 0.5 \\
Finished procedure to left operating room & $3.49(2.87-4.11)$ & $44.52(36.8152 .23)$ & 0.1 \\
Out of operating room to next patient sent for & $8.23(0.45-16)$ & $<0.0001$ \\
\hline
\end{tabular}

Table 3 Prevalence of risk factors for COVID-19 related complications in study population

\begin{tabular}{ll}
\hline Risk factor for COVID-19 complications & $\begin{array}{l}\text { Number } \\
\text { of patients } \\
\text { affected }\end{array}$ \\
\hline Age $>70$ years & 7 \\
Black, Asian or Minority Ethnicity & 0 \\
Hypertension & 12 \\
Ischaemic Heart Disease & 1 \\
Chronic Kidney Disease & 3 \\
Chronic Obstructive Pulmonary Disease or Asthma & 5 \\
Diabetes Mellitus & 1 \\
Malignancy & 1 \\
\hline
\end{tabular}

\section{Patient compliance and attitudes to surgery}

Of the 37 patients included, 19 had no risk factors for complications of COVID-19 whilst 10 had one associated risk factor, four had two associated risk factors and four had three associated risk factors. Table 3 outlines the number of patients affected by each individual risk factor assessed with hypertension being the most commonly encountered within this study population, followed by age greater than 70 years and chronic obstructive pulmonary disease/asthma. Only one of the 37 patients had previously tested positive for COVID-19 on nose and throat swab, however, a total of two patients did believe that they had had COVID-19 previously. All patients tested negative for COVID-19 on nose and throat swab a maximum of $72 \mathrm{~h}$ prior to their procedure. Of the procedures performed during the study period, 19 were minor procedures and 18 were major procedures.

Overall, 31 patients provided information on pre-operative isolation. Of this, 18 patients were fully compliant. Patient-specific failure accounted for four of 13 failures, whilst the remaining nine failures to comply with pre-operative isolation were a result of other members of the household attending either school and/or work during the 14-day isolation period. Sub-group analysis revealed that there was no statistically significant difference between failure rates

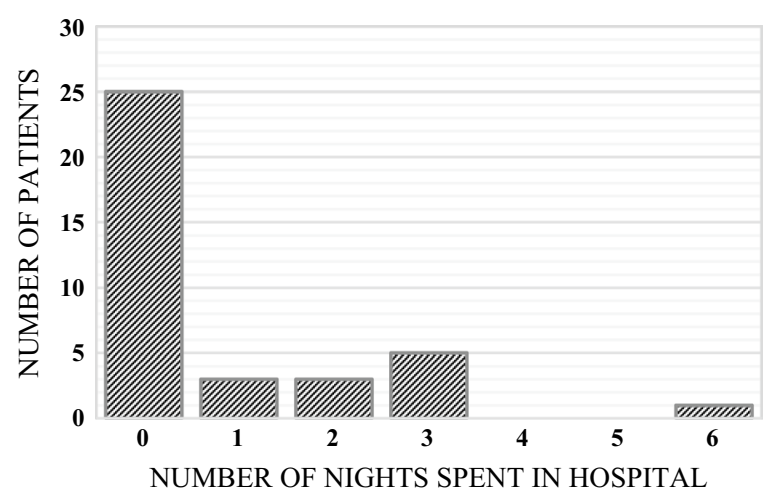

Fig. 2 Length of Hospital Stay. Measured as number of nights spent in hospital

in those with risk factors compared to those without risk factors, with a $p$ value of 0.1 .

Thirty-five patients provided information regarding their attitude towards their upcoming surgery. As a result of the COVID-19 pandemic, 16 patients felt more anxious, whilst 17 felt no different and two felt less anxious. Sub-group analysis of patients revealed that although more patients with risk factors reported increased anxiety towards their surgery compared to those with no risk factors (10 and six, respectively), that this was not statistically significant with a $\mathrm{p}$ value of 0.2 . The impact of the new precautions was mixed with 17 patients describing reduced anxiety and a small proportion stating that the precautions implemented actually led to a slight increase in their anxieties (five patients), whilst the remainder reported no difference (13 patients).

All patients were safely discharged home with 25 of 37 patients being managed as day cases as illustrated in Fig. 2 . Two patients reported minor complications in the post-operative period, both of which were known complications of the relevant procedure (pain and wound leakage). Only one patient had a repeat COVID-19 swab within the 14-day postoperative period, which was negative. This was an asymptomatic screening swab performed due to attendance at hospital for investigation of non-COVID-19 related illness. To the best of our knowledge, no other patients required a repeat 
COVID-19 swab post-operatively. One patient described increased fatigue in the post-operative period, whilst one patient complained of headache but both patients denied any other symptoms of possible COVID-19 infection. All other patients were entirely asymptomatic of COVID-19 infection in the 14 days following surgery.

\section{Discussion}

The COVID-19 pandemic has had an unprecedented impact on public health and economy worldwide. It is projected that the cessation of elective operating in the UK, including orthopaedics, has led to the cancellation of 516,000 operations nationwide over a 12-week period within the first wave of the COVID-19 pandemic [2]. Guidance has since been published by PHE, NHS England and the Surgical Colleges to support the safe re-introduction of elective services [4-6]. However, a second wave of infection brings new challenges as we look forward at the possibility of "a new normal" and what this may entail.

Our data have shown mixed feelings in patients regarding elective surgery with $45.7 \%$ of patients reporting increased anxiety as a result of the COVID-19 pandemic. It is important to note, that despite the increased anxiety, all patients opted to proceed with surgery following the consent process. Unfortunately, we identified patients on admission to hospital and do not have data regarding how many patients declined surgery prior to this. However, Chang et al. performed a study of 102 patients exploring patient's desire for elective surgery following the COVID-19 pandemic and found that $56.8 \%$ would prefer to continue with their planned surgical care on resumption of elective orthopaedic services [2]. This suggests that there remains a significant proportion of patients on the waiting list for elective surgery who would continue with their scheduled care, but for whom their burden of disease and quality of life may continue to deteriorate as their expected date of surgery continues to be delayed.

In fact, waiting times are currently at record highs with 83,203 patients waiting over 52 weeks from referral to treatment in England in July 2020 [7]. This study has highlighted an additional challenge to tackling waiting lists with regards to theatre efficiency. In the Phase three letter from the NHS chief executive, the proposed targets were a return to $80 \%$ of previous activity by September 2020 and to $90 \%$ of previous activity by October 2020 [8]. We found a reduction in theatre efficiency as a result of new infection control procedures which is the equivalent of one minor procedure, such as knee arthroscopy, per session which may pose a challenge to meeting this target. The National Institute for Health and Care Excellence (NICE) has published guidelines on the management of elective patients in light of COVID-19 with proposed methods to increase productivity such as having a standby list of patients in the event that there is a last minute cancellation or where possible utilising two treatment areas to allow one area to be in use whilst the other is cleaned [9]. That said, questions have been raised about the feasibility of a standby list in clinical practice, as this may mean prolonged or indefinite isolation and the possibility of repeated COVID-19 tests as patients await potential dates for surgery. In addition, the surgical collaborative network led by Banghu et al. has also predicted that even if a $10 \%$ increase in baseline surgical activity was achieved, it would unfortunately still take 88 weeks to clear this backlog in the UK [10].

A study by Scott et al. found that $19 \%$ of patients awaiting total hip arthroplasty (THA) and $12 \%$ of patients awaiting total knee arthroplasty (TKA) were considered to be in a health state "worse than death" (WTD) based on their EuroQol five-dimension (EQ-5D) score [2, 11]. Scott et al. also suggested from their findings that increased waiting times may be proportional to the number of patients who are WTD particularly for patients awaiting TKA $[2,11]$, which is alarming given the significantly increased waiting times which have resulted from the pandemic. Furthermore, the economic impact of the temporary cessation of elective surgery is also of significance. Banghu et al. also estimated that it would cost over $£ 2$ billion to clear the backlog of accrued cancelled surgical cases over the first wave of the COVID-19 pandemic [10]. Therefore, it is essential to establish a safe and sustainable framework for the resumption of elective services which is both efficient and deemed acceptable to patients and staff alike.

Coimbra et al. proposed a staged approach to the reintroduction of elective orthopaedic services beginning with assessment of local cases [12]. They suggested that a reduced incidence in community cases and hospital/critical care admissions for a minimum of two weeks, combined with adequate resource availability was required prior to commencing staged re-introduction of elective surgery [12]. This may be of particular relevance as case numbers continue to rise and fall at varying rates over the UK leading to the institution of so-called local lockdowns. Monitoring of local COVID-19 cases, bed capacity including critical care beds, PPE availability, availability of testing and workforce projection is crucial to ensure that elective procedures may continue safely in the event of a local rise in cases $[12,13]$. Timely intervention is key and it may be that temporary suspension of elective services is required at a local level if any concerns are identified. Once safe to do so, re-implementation itself may take different forms depending on hospital infrastructure but the overarching theme should always be the fundamental principle of "do no harm". The majority of the suggested changes have been incorporated into our protocols. A notable exception includes regular COVID-19 testing for staff members [12]. Instead, our policy included 
all trust staff members agreeing to undertake a "self-screening" each day prior to attending their shift for any signs or symptoms of COVID-19 and arranging a test via their line manager if necessary. The staff member would be required to self-isolate and not attend work until the results of the test were available and confirmed negative. To the best of our knowledge, the use of the self-screening protocol and PPE has prevented nosocomial transmission of COVID-19 to the patients included in this study. However, a limitation is that our patients did not undergo routine post-operative COVID-19 testing. That said, this approach has also had no negative implications in other studies which used targeted COVID-19 testing for symptomatic staff members only [14].

The reproducibility of our proposed framework is evidenced in a similar study which was conducted early in the pandemic by Gonzi et al. This study utilised a COVID-19 free site for semi-urgent ambulatory trauma. At the time of the study, routine COVID-19 testing was not available and instead patients were screened for symptoms of COVID19 at presentation and again on admission. All patients included were asymptomatic at the time of follow-up telephone consultation which is largely in keeping with our own results and supports our suggestion that our protocol is a safe approach to providing elective orthopaedic services [14]. That said, Lei et al. conducted a retrospective analysis of patients who were inadvertently listed for surgery in China during the incubation period of COVID-19 and subsequently turned out to be positive for the virus. They found $20.6 \%$ of patients died of COVID-19 related complications with a median time between surgery to onset of symptoms of two days in these patients, all of whom were asymptomatic pre-operatively [15]. Therefore, we would strongly advocate both obtaining negative COVID-19 tests for all patients attending for elective surgery within $72 \mathrm{~h}$ of the planned operation date and also consenting patients for the possibility of false negative results and the implications that this may have for them given the individual circumstances of the patient [4].

One key area in which this framework may require further development is with regards to the pre-operative isolation period. During our study period, the UK government was encouraging the public to work from home if able and was supporting employers with the payment of wages of furloughed employees by paying $80 \%$ of the employee wages up to a pre-defined national cap [16]. Therefore, it is possible that for some patients it was easier, from a financial perspective, to comply with the mandatory pre-operative household isolation. Even with this consideration, our compliance rate was still only 58\%. However, as this scheme is gradually phased out for some, some members of the patient's household may not be able to take time off from work due to financial constraints. This may in turn result in potential inequalities where elective orthopaedic services are not as accessible to patients with low income, which is of particular concern as an association has been shown between social deprivation and WTD state in patients awaiting THA [11]. New NICE guidance suggests that this requirement may be reviewed on a case by case basis and may remove the requirement of 14-day isolation period pre-operatively for some patients provided that they are adhering to the other infection control measures outlined by PHE [8, 9]. Isolation of the patient within their own household may be another potential solution to this problem in the event that pre-operative isolation is advised but that the entire household is unable to isolate, however, the feasibility of this would also have to be assessed.

\section{Limitations}

The significantly high rate of patients declining to participate is of note in this study and led to an overall relatively low number of patients included in the relevant section regarding attitudes and compliance with pre-operative isolation. Of the 58 patients who attended for elective procedures during our study period, 21 declined to take part. In addition, as per Fig. 1, there were a further 3 patients who declined to be contacted for follow-up and 1 patient who was uncontactable via telephone for follow-up consultation. One possible reason for such a significant proportion of patients declining to participate may be through concern that disclosure of non-compliance with pre-operative isolation may have had implications for their impending surgery despite reassurance from the research team.

\section{Conclusion}

Safety of patients undergoing elective orthopaedic surgery remains paramount. Whilst attitudes towards surgery are mixed, the literature suggests that there remains a significant backlog of patients wishing to undergo surgery. However, theatre efficiency has been reduced as a result of new COVID-19 guidelines which will have an impact on national targets to return to near baseline surgical activity. A safe and sustainable framework for the delivery of elective orthopaedic services is required urgently in order to manage this backlog. From our data and review of the literature, we recommend utilisation of COVID-19 free sites wherever possible, appropriate pre-operative risk stratification, informed consent regarding COVID-19 risk, mandatory pre-operative COVID-19 testing within $72 \mathrm{~h}$, daily staff self-screening and adherence to infection control and prevention guidelines with regards to PPE usage and cleaning of clinical areas including the operating theatre. We would advise whole household pre-operative isolation to be assessed and discussed on a case by case basis and further 
measures to improve efficiency to be implemented at the individual hospital level depending on hospital infrastructure and resources.

Authors' Contributions CA designed the study. CA, SS and KN collected data. SS and CA were involved in data analysis and initial preparation of manuscript with all 4 authors contributing to revision of manuscript thereafter.

Data Availability Available on request.

\section{Declarations}

Conflict of interest There are no conflicts of interest to declare.

Ethics Approval Study protocol approved by local audit and governance department.

Consent to Participate and Publication Informed consent obtained from each individual participant.

\section{References}

1. World Health Organisation (2020) Timeline of WHO's response to COVID-19. https://www.who.int/news-room/detail/29-06-2020covidtimeline Accessed 10th August 2020

2. Chang J, Wignadasan W, Kontoghiorghe C et al (2020) Restarting elective orthopaedic services during the COVID-19 pandemic. Bone Joint Open 1(6):267-271. https://doi.org/10.1302/26331462.16.BJO-2020-0057

3. Liow MHL, Tay KXK, Yeo NEM et al (2020) From "business continuity" to "back to business" for orthopaedic surgeons during the COVID-19 pandemic. Bone Joint Open 1(6):222-228. https:// doi.org/10.1302/2633-1462.16.BJO-2020-0036.R1

4. Wallace CN, Kontoghiorghe C, Kayani B, Chang JS, Haddad FS (2020) The impact of COVID-19 on trauma and orthopaedic surgery in the United Kingdom. Bone Joint Open 1(7):420-423. https://doi.org/10.1302/2633-1462.17.BJO-2020-0102

5. NHS England (2020) Operating framework for urgent and planned services in hospital settings during COVID-19. https://www.engla nd.nhs.uk/coronavirus/wp-content/uploads/sites/52/2020/05/ Operating-framework-for-urgent-and-planned-services-withinhospitals.pdf Accessed 10th August 2020

6. National Health Service (2020) Who's at risk from coronavirus. https://www.nhs.uk/conditions/coronavirus-covid-19/ people-at-higher-risk/whos-at-higher-risk-from-coronavirus/ Accessed 10th August 2020

7. NHS England and NHS Improvement (2020) Statistical Press Notice, NHS referral to treatment (RTT) waiting times data July 2020. https://www.england.nhs.uk/statistics/wp-content/uploads/ sites/2/2020/09/Jul20-RTT-SPN-publication-v0.pdf Accessed 10th August 2020

8. Stevens S, Pritchard A (2020) NHS Chief Executive's Phase 3 Letter. https://www.england.nhs.uk/coronavirus/wp-content/uploads/ sites/52/2020/07/Phase-3-letter-July-31-2020.pdf Accessed 10th August 2020

9. National Institute for Health and Care Excellence (2020) COVID19 rapid guideline: arranging planned care in hospitals and diagnostic services. https://www.nice.org.uk/guidance/ng179 Accessed 11th September 2020

10. COVIDSurg Collaborative (2020) Elective surgery cancellations due to the COVID-19 pandemic: global predictive modelling to inform surgical recovery plans. Br J Surg. https://doi.org/10.1002/ bjs. 11746

11. Scott CEH, MacDonald DJ, Howie CR. (2019) "Worse than death" and waiting for a joint arthroplasty. Bone Joint J. 101B(8): 941-950 https://doi.org/10.1302/0301-620X.101B8.BJJ2019-0116.R1

12. Coimbra R, Edwards S, Coimbra BC, Tabuenca A. (2020) Resuming elective surgical services in times of COVID-19 infection. Trauma Surg Acute Care Open. 5:e000511. https://doi.org/10. 1136/tsaco-2020-000511

13. Ding BTK, Tan KG, Oh JYL, Lee KT (2020) Orthopaedic surgery after COVID-19 - A blueprint for resuming elective surgery after a pandemic. Int J Surg 80:162-167. https://doi.org/10.1016/j. eclinm.2020.100331

14. Gonzi G, Rooney K, Gwyn R et al (2020) Trauma surgery at a designated COVID-19-free site during the pandemic. Bone Joint Open 1(6):302-308. https://doi.org/10.1016/j.eclinm.2020.100331

15. Lei S, Jiang F, Su W et al (2020) Clinical characteristics and outcomes of patients undergoing surgeries during the incubation period of COVID-19 infection. EClinicalMedicine 21:100331. https://doi.org/10.1016/j.eclinm.2020.100331

16. HM Revenue and Customs (2020) Changes to the Coronavirus Job Retention Scheme. https://www.gov.uk/government/publicatio ns/changes-to-the-coronavirus-job-retention-scheme/changes-tothe-coronavirus-job-retention-scheme Accessed 10th August 2020

Publisher's Note Springer Nature remains neutral with regard to jurisdictional claims in published maps and institutional affiliations. 